\title{
Some Remarks to Numerical Solutions of the Equations of Mathematical Physics
}

\author{
Ludmila Petrova \\ Department of Computational Mathematics and Cybernetics, Moscow State University, Moscow, Russia \\ Email: ptr@cs.msu.su
}

Received May 16, 2013; revised June 25, 2013; accepted July 7, 2013

Copyright (C) 2013 Ludmila Petrova. This is an open access article distributed under the Creative Commons Attribution License, which permits unrestricted use, distribution, and reproduction in any medium, provided the original work is properly cited.

\begin{abstract}
The equations of mathematical physics, which describe some actual processes, are defined on manifolds (tangent, a companying or others) that are not integrable. The derivatives on such manifolds turn out to be inconsistent, i.e. they don't form a differential. Therefore, the solutions to equations obtained in numerical modelling the derivatives on such manifolds are not functions. They will depend on the commutator made up by noncommutative mixed derivatives, and this fact relates to inconsistence of derivatives. (As it will be shown, such solutions have a physical meaning). The exact solutions (functions) to the equations of mathematical physics are obtained only in the case when the integrable structures are realized. So called generalized solutions are solutions on integrable structures. They are functions (depend only on variables) but are defined only on integrable structure, and, hence, the derivatives of functions or the functions themselves have discontinuities in the direction normal to integrable structure. In numerical simulation of the derivatives of differential equations, one cannot obtain such generalized solutions by continuous way, since this is connected with going from initial nonintegrable manifold to integrable structures. In numerical solving the equations of mathematical physics, it is possible to obtain exact solutions to differential equations only with the help of additional methods. The analysis of the solutions to differential equations with the help of skew-symmetric forms [1,2] can give certain recommendations for numerical solving the differential equations.
\end{abstract}

Keywords: Two Systems of Reference; Nonintegrable Manifolds and Integrable Structures; Solutions of Two Types; Discrete Transitions; Observable Formations

\section{Specific Features of Solutions to Equations Describing Actual Processes}

Let us take the simplest case: the first-order partial differential equation

$$
F\left(x^{i}, u, p_{i}\right)=0, \quad p_{i}=\partial u / \partial x^{i}
$$

The exact solution (the solution that depends only on variables, i.e. it is a function) can be obtained in the case when the derivatives obeying the equations made up the differential.

Let us construct the differential made up of derivatives that obey the differential equation

$$
\mathrm{d} u=\theta
$$

where $\theta=p_{i} \mathrm{~d} x^{i}$ (the summation over repeated indices is implied). It should be noted that $\theta=p_{i} \mathrm{~d} x^{i}$ is a skewsymmetric differential form of the first degree.

It appears that, in the general case when differential Equation (1) describes any physical processes, the form $\theta=p_{i} \mathrm{~d} x^{i}$ made up of derivatives of differential equation is not a differential.

For the form $\theta=p_{i} \mathrm{~d} x^{i}$ be a differential, its differenttial has to be equal to zero.

The differential $\mathrm{d} \theta$ of the form $\theta=p_{i} \mathrm{~d} x^{i}$ can be written as $K_{i j} \mathrm{~d} x^{i} \mathrm{~d} x^{j}$, where

$$
K_{i j}=\partial p_{j} / \partial x^{i}-\partial p_{i} / \partial x^{J}
$$

are the components of the differential form commutator. From Equation (1) it does not follow (explicitly) that the derivatives $p_{i}=\partial u / \partial x^{i}$, which obey to the equation (and to given boundary or initial conditions), are consistent, that is, their mixed derivatives are commutative. The components of the commutator $K_{i j}$ is nonzero. Therefore, the differential form commutator and the differenttial of the form $\theta$ are nonzero. This points to the fact that the differential expression made up of the derivatives of differential equation is not a differential. That is, the derivatives of differential equation do not made up a dif- 
ferential (without additional conditions).

This means that the solution $u$ to Equation (1) obtained from such derivatives is not a function of variables $x^{i}$ only. This solution will depend on the commutator $K_{i j}$ with nonzero value related to inconsistence of derivatives.

To obtain the solution that is a function (i.e., the derivatives of this solution made up a differential), it is necessary to add the closure condition (vanishing the form differential) for the form $\theta=p_{i} \mathrm{~d} x^{i}$ and for the relevant dual form (in the present case the functional $F$ plays a role of the form dual to $\theta$ ):

$$
\left\{\begin{array}{l}
\mathrm{d} F\left(x^{i}, u, p_{i}\right)=0 \\
\mathrm{~d}\left(p_{i} \mathrm{~d} x^{i}\right)=0
\end{array}\right.
$$

If to expand the differentials, one gets a set of homogeneous equations with respect to $\mathrm{d} x^{i}$ and $\mathrm{d} p_{i}$ (in the $2 n$-dimensional space):

$$
\left\{\begin{array}{l}
\left(\partial F / \partial x^{i}+p_{i} \partial F / \partial u\right) \mathrm{d} x^{i}+\mathrm{d} p_{i} \partial F / \partial p_{i}=0 \\
\mathrm{~d} p_{i} \mathrm{~d} x^{i}-\mathrm{d} x^{i} \mathrm{~d} p_{i}=0
\end{array}\right.
$$

It is well known that vanishing the determinant composed of coefficients at $\mathrm{d} x^{i}, \mathrm{~d} p_{i}$ is the solvability condition of the set of homogeneous differential equations. This leads to relations:

$$
\frac{\mathrm{d} x^{i}}{\partial F / \partial p_{i}}=\frac{-\mathrm{d} p_{i}}{\partial F / \partial x^{i}+p_{i} \partial F / \partial u}
$$

Relations (4) specify the integrating direction, which defines an integrable structure, that is, a pseudostructure (in its metric properties) on which the form $\theta=p_{i} \mathrm{~d} x^{i}$ turns out to be closed one, i.e. this form becomes a differential.

On the pseudostructure, which is defined by relation (4), the derivatives of differential Equation (1) constitute the differential $\delta u=p_{i} \mathrm{~d} x^{i}=\mathrm{d} u$ (on the pseudostructure), and this means that the solution to Equation (1) becomes a function. Solutions, namely, functions on the pseudostructures, are so-called generalized solutions. The characteristics, characteristic surfaces, singular points, potential surfaces, and others are examples of pseudostructures or their formations.

It should be underlined the following. The solutions, which are functions, are obtained only under additional condition that determines integrable structures. This additional condition, as one can see, is vanishing the determinant. Such an additional condition is a condition of degenerate transformation. The degenerate transformation executes the transition from tangent nonintegrable manifold of differential equations to integrable structures (pseudostructures). (The Legendre transformations are examples of such a transformation.) The realization of ad- ditional conditions (that can be caused by any degree of freedom) leads to the realization of integrable structure and the transition from the solutions, which are not functions, to generalized solutions (functions).

One can see that the generalized solutions cannot be obtained by numerical modelling the differential equation only on original tangent manifold.

The first-order partial differential equation has been analyzed. Similar functional properties have all differential equations describing actual processes. Below it will be shown that the sets of differential equations that describe actual processes possess such properties.

\section{The Properties and Peculiarities of Solutions to the Equations of Mechanics and Physics of Continuous Medium}

The equations of mechanics and the physics of continuous media (material systems such as gas-dinamical and cosmological systems, the systems of charged particles and others) are the equations that describe conservation laws for energy, linear momentum, angular momentum, and mass [3] (The set of Navier-Stokes equations is an example [3]). Such conservation laws can be referred to as the balance conservation laws since these laws establish a balance between variations of physical quantities and appropriate external action.

The equations of conservation laws are differential (or integral) equations that describe the variation of functions corresponding to physical quantities like the particle velocity (of elements), temperature or energy, pressure and density. Since these physical quantities relate to one material system, the connection between them has to exist. This connection is described by state functional that specifies the material system state. The action functional, entropy, the Pointing vector, Einstein's tensor, wave function and others can be regarded as examples of such functionals [4]. From the equations of conservation law, it follows the evolutionary relation for state functional, which enables one to disclose the properties and peculiarities of the solutions to the equations of mechanics and the physics of continuous media.

\subsection{Evolutionary Relation}

When studying the solutions to partial differential equations the conjugacy of derivatives with respect to various variables was analyzed. When describing physical processes in continuous media (in material systems) one obtains not one differential equation but a set of differential equations. And in this case it is necessary to investigate the conjugacy of not only derivatives but also the conjugacy (consistency) of the equations of this set.

The equations are consistent if they can be contracted into identical relations for the differentials, i.e. for closed forms. 
Let us now analyze the consistency of the equations that describe the conservation laws for energy and linear momentum.

In the accompanying frame of reference, which is tied to the manifold built by the trajectories of particles (elements of material system), the equation for energy is written in the form (see example [5])

$$
\frac{\partial \psi}{\partial \xi^{1}}=A_{1}
$$

Here $\xi^{1}$ are the coordinates along the trajectory, $\psi$ is the functional of the state, $A_{1}$ is the quantity that depends on specific features of the material system and external (with respect to the local domain) energy actions onto the system.

Similarly, in the accompanying frame of reference, the equation for linear momentum appears to be reduced to the equation of the form

$$
\frac{\partial \psi}{\partial \xi^{v}}=A_{\nu}, \quad v=2, \cdots
$$

where $\xi^{v}$ are the coordinates along the direction normal to the trajectory, $A$, are the quantities that depend on the specific features of material system and the external force actions.

Equations (5) and (6) can be convoluted into the relation

$$
\mathrm{d} \psi=A_{\mu} \mathrm{d} \xi^{\mu} \quad(\mu=1, v)
$$

where $\mathrm{d} \psi$ is the differential expression

$\mathrm{d} \psi=\left(\partial \psi / \partial \xi^{\mu}\right) \mathrm{d} \xi^{\mu}$.

Relation (7) can be written as

$$
\mathrm{d} \psi=\omega
$$

here $\omega=A_{\mu} \mathrm{d} \xi^{\mu}$ is a skew-symmetric differential form of the first degree.

[In the case of the Euler and Navier-Stokes equations a concrete form of relation (8) and its properties were considered in papers [5]].

Since the equations of balance conservation laws are evolutionary ones, the relation obtained is also an evolutionary relation.

Relation (8) was obtained from the balance conservation law equations for energy and linear momentum. In this relation the form $\omega$ is that of the first degree. If the balance conservation law equation for angular momentum be added to the equations for energy and linear momentum, this form in the evolutionary relation will be a form of the second degree. And in combination with the equation of the balance conservation law for mass this form will be a form of degree 3 .

Thus, in the general case, the evolutionary relation can be written as

$$
\mathrm{d} \psi=\omega^{p}
$$

where the form degree $p$ takes the values $p=0,1,2,3$.

Evolutionary relation obtained from the equations of the balance conservation laws possesses some peculiarity. This relation proves to be nonidentical since the differential form in the right-hand side of this relation is not a closed form, and, hence, this form cann't be a differential like the left-hand side.

Let us analyse the relation (8).

The evolutionary relation $\mathrm{d} \psi=\omega$ is a nonidentical relation as it involves the unclosed skew-symmetric differential form $\omega=A_{\mu} \mathrm{d} \xi^{\mu}$. The form $\omega$ isn't a close form since its differential $\mathrm{d} \omega$ is nonzero. The differential $\mathrm{d} \omega$ can be written as $K_{\alpha \beta} \mathrm{d} \xi^{\alpha} \mathrm{d} \xi^{\beta}$, where

$$
K_{\alpha \beta}=\partial A_{\beta} / \partial \xi^{\alpha}-\partial A_{\alpha} / \partial \xi^{\beta}
$$

are the components of the differential form commutator built of the mixed derivatives (here the term connected with the nonintegrability of the manifold has not yet been taken into account). The coefficients $A_{\mu}$ of the form $\omega$ can be obtained either from the equations of the balance conservation law for energy or from that for linear momentum. This means that in the first case the coefficients depend on the energetic action and in the second case they depend on the force action. In actual processes energetic and force actions have different nature and appear to be inconsistent. The commutator of the form $\omega$ constructed from the derivatives of such coefficients is nonzero. This means that the differential of the form $\omega$ is nonzero as well. Thus, the form $\omega$ proves to be unclosed and cannot be a differential.

[The skew-symmetric form in evolutionary relation is defined on the manifold made up by trajectories of the material system elements. Such a manifold is a deforming manifold. The commutator of the skew-symmetric form defined on such manifold includes an additional term connected with the differential of the basis. This term specifies the manifold deformation and hence is nonzero. Both terms in the commutator (obtained by differentiating the basis and the form coefficients) have a different nature and, therefore, cannot compensate one another. This fact once more emphasize that the evolutionary form commutator, and, hence, its differential, are nonzero. That is, the evolutionary form remains to be unclosed].

Hence, without the knowledge of a particular expression for the form $\omega$, one can argue that for actual processes the evolutionary relation proves to be nonidentical.

The nonidentity of the evolutionary relation means that the initial equations of conservation laws turn out to be inconsistent, and hence, they are not integrable. The solutions to these equations will not be functions without additional conditions. They will depend on the commutator, which is nonzero due to inconsistence of the conservation law equations. 
The solutions that are functions can be obtained only under additional conditions when the identical relation can be obtained from nonidentical evolutionary relation. This will point to the consistency (but only local, under additional condition) of the conservation law equations and the local integrability.

The identical relation can be obtained from nonidentical evolutionary relation only in the case when the closed exterior form, which is a differential, is obtained from unclosed evolutionary form. This is possible only under degenerate transformation, namely, under the transformation that does not conserve the differential, since, the evolutionary form differential is nonzero, whereas the differential of closed form is equal to zero. The additional conditions (which are the conditions of local integrability) are the conditions of degenerate transformation.

The additional conditions are caused by any degrees of freedom. The vanishing of functional expressions such as the determinant, Jacobian and so on corresponds to the additional conditions. These conditions can be realized under changing the evolutionary relation, which is selfvariable.

If the conditions of degenerate transformation are realized, from the unclosed evolutionary form with nonvanishing differential $\mathrm{d} \omega^{p} \neq 0$, one can obtain the differential form closed on pseudostructure. The differential of this form equals zero. That is, it is realized the transition

$$
\mathrm{d} \omega^{p} \neq 0 \rightarrow(\text { degenerate transformation }) \rightarrow\left\{\begin{array}{l}
\mathrm{d}_{\pi} \omega^{p}=0 \\
\mathrm{~d}_{\pi}^{*} \omega^{p}=0
\end{array}\right.
$$

where ${ }^{*} \omega^{p}$ is the dual form (which is a metric form). The condition $\mathrm{d}_{\pi}^{*} \omega^{p}=0$ is an equation for a certain pseudostructure $\pi$ on which the differential of evolutionary form vanishes: $\mathrm{d}_{\pi} \omega^{p}=0$. This points to the fact that the pseudostructure is realized, and the closed (inexact) exterior form $\omega_{\pi}^{p}$ is obtained on pseudostructure.

On the pseudostructure, from evolutionary relation $\mathrm{d} \psi=\omega^{p}$ it is obtained the identical relation $\mathrm{d}_{\pi} \psi=\omega_{\pi}^{p}$, since the closed exterior form $\omega_{\pi}^{p}$ is a differential of some differential form. (This relation will be an identical one as the left and right sides of the relation contain differentials). The identity of the relation obtained from the evolutionary relation means that on pseudostructures the original equations for material system (the equations of conservation laws) become consistent and integrable.

Pseudostructures constitute the integrable surfaces (such as characteristics, singular points, potentials of simple and double layers, and others) on which the quantities of material system desired (such as the temperature, pressure, density) become functions of only independent variables and do not depend on the commutator (and on the path of integrating). This are generalized solutions. They may be found by means of integrating (on integradle structures) the equations of conservation laws for material systems.

Since generalized solutions are defined only on realized integrable structures (pseudostructures), they or their derivatives have discontinuities in the direction normal to integrable structure [6].

One can see that the integrable structures are obtained from the condition of degenerate transformation of the evolutionary relation. The conditions of degenerate transformation (a vanishing of such functional expressions as determinants, Jacobians, Poisson's brackets, residues) are connected with the symmetries, which can be due to the degrees of freedom of the material systems under consideration (for example, the translational, rotational and oscillatory degrees of freedom of material system).

The degenerate transformation is realized as the transition from the noninertial frame of reference to the locally inertial one, i.e. the transition from nonintegrable manifold (for example, tangent or accompanying) to integrable structures and surfaces.

Thus, one can see that the solutions to the set of equations, as well as in the case of a single equation, may be of two types: the solutions that are not functions, that is, they depend not only on independent variables, and generalized solutions, which are functions, and are obtained only under realization of additional conditions (which determine integrable structures or surfaces). The specific feature is the fact that they are definded on different spatial objects. Such solutions cannot be obtained by continuous modelling the differential equations only on a single spatial odject.

Before turning back to the problems of numerical solving the differential equations, it should call attention to the physical meaning of the solutions to these equations.

\subsection{Physical Meaning of the Solutions to the Mathematical Physics Equations}

The physical meaning of the solutions to the mathematical physics equations can be understood by the analysis of the evolutionary relation.

The evolutionary relation includes the functional that specifies the system state. Sinse this relation is nonidentical, it is impossible to obtain the state functional from this relation. This points to the absence of the state function and nonequilibrium state of the material system under consideration. The solutions of the first type just describe such nonequilibrium state. In this case, the commutator describes the internal force that induces the nonequilibrium state of material system. The solutions of the second type (genealized solutions, which are functions) are obtained under realization of additional conditions 
when the closed exterior forms is obtained from the unclosed evolutionary form, and the identical relation is realized. From such relation one can get the state functional and find the state function. This fact will point to the transition of material system into the locally equilibrium state.

The transition of the material system from nonequilibrium state into the locally-equilibrium one means that the unmeasurable quantity described by the nonzero commutator of the unclosed evolutionary differential form, which acts as an internal force, transforms into the measurable quantity. In material system, this reveals as the emergence of certain observable formations, which develop spontaneously. Such formations and their manifestations are fluctuations, turbulent pulsations, waves, vortices, and others [7].

It appears that the transition from the solutions of the first type to the generalized solution corresponds to the transition of material system from the nonequilibrium state to the locally equilibrium one that is accompanied by the emergence of a certain (observable) formation in material system. The discontinuous functions that correspond to generalized solutions just describe such observable formations.

Thus we obtain that the discrete realization of generalized solution points to the emergence of a certain (observable) formation in material system that is described by discontinuous functions corresponding to generalized solutions.

It may be also noted that the type of solutions to the equations describing material systems is of great significance for mechanics and physics of continuous media. In mechanics and physics of continuous media the same equations are considered (the equations of conservation laws for energy, linear momentum, angular momentum, and mass). The set of Navier-Stokes equations is an example [5]. However, the approaches to solving these equations in mechanics and physics are different. In physics the interest is expressed in only generalized solutions that are invariant ones and describe measurable physical quantities (but not the process itself), and noninvariant solutions are ignored (even if they have a physical meaning). The aim of mechanics of continuous media is to describe the process of the continuous media evolution. And in this case the numerical methods of solving differential equations are commonly used without studying the integrability conditions of these equations. The question of searching for invariant solutions that are realized only under additional conditions is commonly not posed. That is, one considers the solutions that are not functions.

Such restricted approaches, both in physics and mechanics, lead to nonclosure of relevant theories and this has some negative points. In mechanics without finding the generalized solutions it is impossible to describe such processes as the emergence of vorticity, turbulence and others. The physical approach enables one to find allowed invariant solutions, however, in this approach there is no way to say at what time instant of evolutionary process one or another exact solution was realized. This does not also discloses the causality of phenomenon described by these solutions. It is evident that in mechanics, as well in physics, it is necessary to seek for solutions of both types. In particular, in the case of gas-dynamic system such an approach had been studied in paper [5].

\section{On the Problem of Numerical Solving the Differential Equations}

As it was noted, the equations that describe actual processes are definded on manifolds (tangent, accompanying), which are nonintegrable. If to model the equations on such a manifold, one can obtain, without additional conditions, the solutions of only first type, i.e. the solutions that depend on the commutator with nonzero value caused by inconsistency of derivatives or equations in the set of equations. It should be emphasized once more that such solutions have physical meaning, namely, they describe the nonequilibrium state induced by the physical processes proceeded in the system. The generalized solutions, which are functions and describe discrete formations, cannot be obtained by modelling the equations only on original manifold, since they are obtained on integrable structures that do not belong to original nonintegrable manifold. Therefore, to obtain the generalized solutions by numerical simulation, one must use two systems of reference. One more problem of obtaining the generalized solution relates to the fact that the integrable structures with generalized solution are not initially given, and they are realized spontaneously in the process of integrating under the realization of additional conditions, namely, the integrability conditions. (As additional conditions it may serve, for example, the characteristic conditions, the dynamical conditions of the consistency of equations in the set of equations [8] and so on). To obtain the integrable structures, it is necessary to trace for the realization of additional conditions, which define the integrable structures, in the process of numerical integrating the equations on the original manifold. This gives a possibility to obtain the instant of realization the generalized solution.

In this case, the transitions from inexact solutions to generalized ones describe the process of emergence of any observable formations (in particular, such as waves, vorticity and others), which intensity is definded by generalized solution.

As it was noted, in mechanics and physics the interest is expressed in various types of the solutions to equations. The methods of numerical solving the equations relate to 
this fact.

In mechanics this is the method of direct numerical simulating the equations, which is fulfilled on tangent manifold (being nonintegrable) and enables one to obtain only inexact solutions.

In physics this is the method of solving equations when the equations are provided with the integrability conditions (the conditions of consistency) and this enables one to obtain integrable structures or surfaces, that is, to go out onto cotangent integrable manifold and obtain exact solutions (the methods of characteristics, symmetries, eigen-functions and others are examples of such methods). The analytical methods may be such methods.

It is possible that the integration of these both methods will allow solve some problems on numerical integrating the mathematical physics equations.

It should be emphasized that on integrable structures the variables of the function desired do not coincide with the variables of original manifold (since they belong to different spatial objects). Thus, the coordinates of the equations for characteristics are not identical to the independent coordinates of the initial manifold, on which the initial equation is defined.

It should be emphasized once more that the existence of two types of solutions has a deep physical sense. This peculiarity of the equations enables one to describe the process of origin of discrete formations such as waves, vorticities, turbulent pulsations [7] and so on.

It has been shown that the origin of discrete formations is described by the transition from the solutions of the first type, which depend on a certain commutator, to the generalized solution that is a function. To describe the process of origin of discrete formations, it is necessary, firstly, have the solutions of the first type, which can be obtained only by numerical modelling of the equation on the original nonintegrable manifold (it is impossible to find such a solution by analytical method), and, secondly, have the solution of the second type (generalized solution). This solution can be obtained only on integrable structure that is definded by the integrability conditions being realized. Here there is a delicate point. The allowable generalized solutions can be obtained by analytical methods if the integrability conditions are imposed on the equations. However, in this case it is impossible to define the instant of realization of generalized solution and thereby to describe the process of the discrete formation emergence. The description of evolutionary processes is possible only either by numerical methods, but with two frames of reference, or by using simultaneously numerical and analytical methods.

\section{Conclusions}

It has been shown that the equations, which describe actual processes, have the solutions of two types, which are defined on different spatial objects, and, therefore, cannot be obtained by continuous numerical simulations of the equations. The existence of two spatial objects on which the solutions are defined gives rise to the problems on which the attention must be focused while numerical solving the mathematical physics equations.

It should be also emphasized that the methods of numerical solving the equations with account for the existence of two types of solutions can allow describing the evolutionary processes such as the emergence of any discrete formations.

\section{REFERENCES}

[1] L. I. Petrova, "Exterior and Evolutionary Differential Forms in Mathematical Physics: Theory and Applications," Lulu.com, 2008, 157 p.

[2] L. I. Petrova, "Role of Skew-Symmetric Differential Forms in Mathematics," 2010. http://arxiv.org/abs/1007.4757

[3] J. F. Clarke and M. Machesney, "The Dynamics of Real Gases," Butterworths, London, 1964.

[4] L. I. Petrova, "Physical Meaning and a Duality of Concepts of Wave Function, Action Functional, Entropy, the Pointing Vector, the Einstein Tensor," Journal of Mathematics Research, Vol. 4, No. 3, 2012, pp. 78-88.

[5] L. I. Petrova, "Integrability and the Properties of Solutions to Euler and Navier-Stokes Equations," Journal of Mathematics Research, Vol. 4, No. 3, 2012, pp. 19-22. doi:10.5539/jmr.v4n3p19

[6] L. I. Petrova, "Relationships between Discontinuities of Derivatives on Characteristics and Trajectories," Journal of Computational Mathematics and Modeling, Vol. 20, No. 4, 2009, pp. 367-372. doi:10.1007/s10598-009-9043-0

[7] L. I. Petrova, "The Noncommutativity of the Conservation Laws: Mechanism of Origination of Vorticity and Turbulence," International Journal of Theoretical and Mathematical Physics, Vol. 2, No. 4, 2012, pp. 84-90. doi:10.5923/j.ijtmp.20120204.05

[8] V. I. Smirnov, "A Course of Higher Mathematics, V. 4," Technology and Theory in the Literature, Moscow, 1957. (in Russian) 